\title{
Successful management of perimortem cesarian section with two types of pelvic packing; a case report
}

\author{
Helvacioglu Caglar ${ }^{*}$, Boukari Bako Bibata ${ }^{1}$, Serdar Karakuzu ${ }^{2}$, Ali Emre Cetinkol ${ }^{3}$, \\ Nursen Atasoy ${ }^{4}$ \\ ${ }^{1}$ TURKISH NIGER FRIENDSHIP HOSPITAL, DEPARTMENT OF OBSTETRICS AND GYNAECOLOGY, NIAMEY, NIGER \\ ${ }^{2}$ TURKISH NIGER FRIENDSHIP HOSPITAL, DEPARTMENT OF ANESTHESIA AND REANIMATION, NIAMEY, NIGER \\ ${ }^{3}$ TURKISH NIGER FRIENDSHIP HOSPITAL, DEPARTMENT OF PEDIATRICS, NIAMEY, NIGER \\ ${ }^{4}$ TURKISH NIGER FRIENDSHIP HOSPITAL, DEPARTMENT OF UROLOGY, NIAMEY, NIGER
}

\section{AB S TR ACT}

Perimortem cesarean is rare and one of the worst possible scenarios in obstetrics. Multidisciplinary approach and speed are extremely important. Pelvic packing in massive postpartum hemorrhages is a method which obstetricians do not commonly use. The patient who had cardiac arrest during travail was successfully managed with a peripartum hysterectomy and two different types of pelvic packing after perimortem cesarean. The mother and baby were healthily discharged. Fetal and maternal survival after perimortem cesarean is quite low. The most important factor determining survival is speed. Pelvic packing is effective in postpartum unstoppable bleeding.

\section{Category: Case Presentation}

Received: December 17, 2020

Accepted: February 11, 2021

Published: May 10, 2021

Keywords:

Cardiac arrest, Perimortem cesarean section, Pregnancy, Pelvic Packing, Bakri balloon tamponade

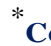

Corresponding author:

Helvacioglu Caglar,

Turkish Niger Friendship Hospital, Department of Obstetrics and Gynecology, Niamey, Niger, Boulevard Mali Bero NO:70 Niamey/ Niger E-mail: caglarhel@ hotmail.com

\section{Introduction}

Cardiopulmonary arrest, rarely seen during a pregnancy, is one of the worst obstetric scenarios to be encountered during travail or in the emergency room [1]. Decision-making about the timing and place of delivery, investigations and intensive care management can be challenging. The term "perimortem" caesarean section is applied to cases where the mother is on cardiopulmonary resuscitation before the caesarean section and shows no signs of recovery afterwards. Maternal, and infant survival after caesarean section performed during cardiopulmonary resuscitation is extremely rare [2].

Obstetric hemorrhage is still the greatest cause of maternal death and reported mortality rates from this disorder is $13.6 \%$ in low-income [3]. Pharmacological treatment, surgical vascular ligations and sutures can be lifesaving in cases of bleeding which do not stop despite selective arterial embolization. Pelvic packing, which obstetricians are not familiar with today is especially effective in stopping raw surface bleeding, venous bleeding, or bleeding in inaccessible locations. It may also be preferred in patients referred to the tertiary center [3].

We herein present the management of a 33-year-old patient who had a cardiac arrest while in our maternity ward with limited facilities in Niger and who underwent peripartum hysterectomy and pelvic packing after the perimortem cesarean delivery (PMCD), along with the healthy discharge of both the mother and the baby afterwards.

\section{Case Report}

A 33-year-old G3P2 woman was hospitalized to the maternity ward at the 39th gestational week with a cephalic presentation and $4 \mathrm{~cm}$ cervical dilatation. She had undergone two previous vaginal deliveries with no complications. Her blood pressure was $130 / 80 \mathrm{mmHg}$, urinary analysis showed $1+$ proteinuria, her platelet count was $313.000 / \mathrm{mL}$. The liver function tests, and other laboratory parameters were within normal limits. The patient who developed an eclamptic convulsion was given MgSO4 at loading dose. She had a cardiopulmonary arrest 
in the postictal period and CPR was started immediately. The time between the onset of CPR and the birth of the baby was 3 minutes, while cardio pulmonary resuscitation (CPR) was performed for a total of 5 minutes. A male fetus weighing $3600 \mathrm{~g}$ was birthed, and his APGAR scores at the first and fifth minutes were 1 and 8, respectively. During PMCD, a postpartum hemorrhage caused by uterine atony that was responsive to uterotonics and disseminated intravascular coagulation (DIC) were noted.

Despite the use of uterotonics and intrauterine Bakri balloon application following the cesarean section, who had continuous vaginal bleeding, hemoglobin value dropped to $3 \mathrm{~g} / \mathrm{dL}$ and platelet decreased to $65,000 / \mathrm{mL}$. The shock index was 1.9. Relaparotomy was performed and uterine arteries were ligated, and since she was unstable, peripartum hysterectomy was initiated without ligation of the hypogastric arteries. After the hysterectomy, hemorrhage continued to fill the pelvis from the peripheral edges. 4 pieces of $30 \times 30 \mathrm{~cm}$ compresses were attached to each other and pelvic packing was performed so that traction could be done from the vaginal cuff and the abdomen was closed.

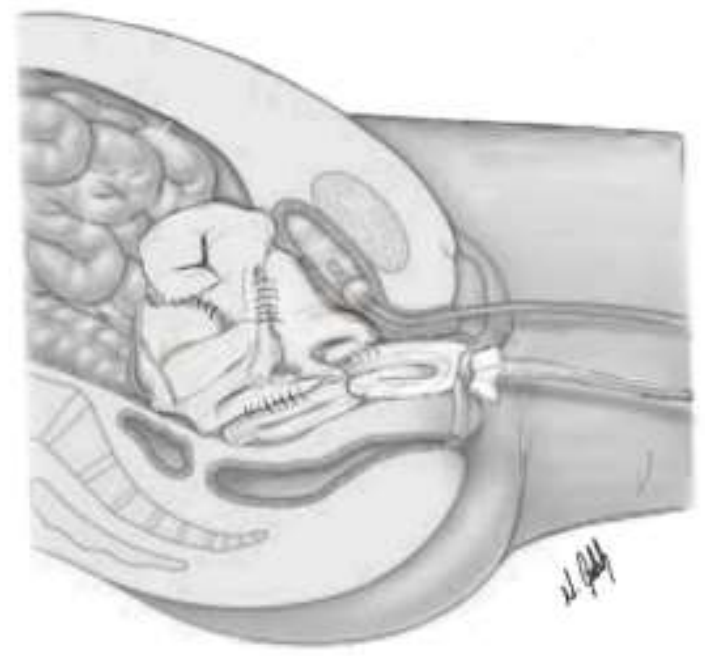

Figure 1. The pelvic packing approach: four compresses attached, and vaginal traction done.

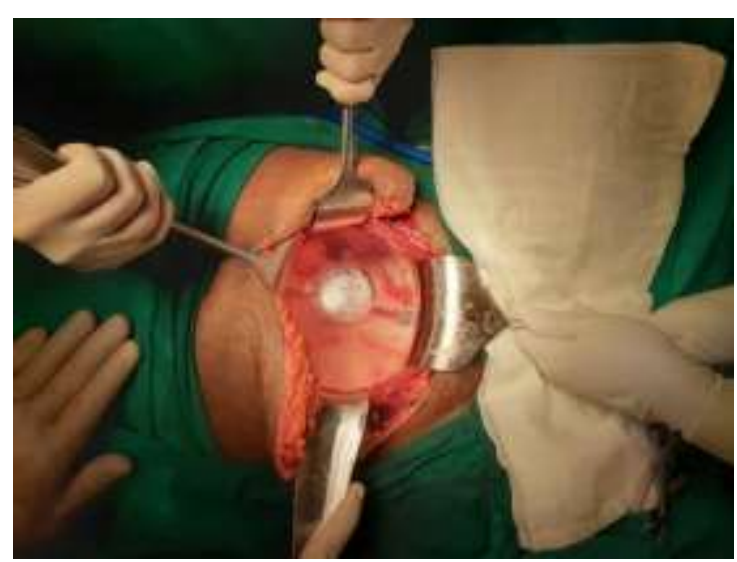

Figure 2: The package with the Bakri balloon within the surgical field. The balloon is inflated with $800 \mathrm{ml}$ sterile water.
After the PEAK pressure increased to $45 \mathrm{mmHg}$ at the 28th hour, a third laparotomy was performed to remove the compress packing (Figure 1). Non-dense adhesions of the small intestines were observed on the compress and gently separated with hydrodissection. Although decreased, blood still pooled in the pelvis. This time, packing was performed with a Bakri Postpartum Balloon (Cook Medical, Bloomington, IN), which was inflated with $800 \mathrm{ml}$ and the abdomen was closed (Figure 2). 20 hours later, the balloon volume was decreased in half, and 6 hours later, it was removed transvaginally.

Due to discharge from the incision site and fat necrosis, scar revision was performed twice. She was discharged from the hospital on the 27 th postoperative day. A total of 10 units of fresh whole blood and 2 units of fresh frozen plasma were administered. No other blood products could be given due to low resource.

\section{Discussions}

Perimortem cesarean delivery is a condition with high feto-maternal mortality and morbidity. The team's experience, timing, and ability to manage stress are crucial to success. It requires intensive care and close monitoring afterwards. According to the American Heart Association, the probability of sequela remaining in the baby increases if CPR lasts longer than 5 minutes [4]. Therefore, speed in perimortem cesarean is particularly important. We preferred Pfannenstiel incision, which we are accustomed to, but similar cases in the literature were approached with a classic midline incision [5].

Obstetric Pelvic packing is a life-saving technique that has been abandoned due to the surgical and interventional radiological techniques developing today. There are varieties such as Umbrella pack using laparotomy pads or roller gauze, Foley catheter - condom tamponade and Bakri balloon [3]. Pelvic packing can be used where hemorrhage persists from large raw surfaces, venous plexuses, and inaccessible areas but obstetricians and we have limited experiences. We have limited data about when to remove, from which route to remove and the use of antithrombolytic therapy [6].

The advantages of compress packing include high availability and accessibility, decreased cost, and effectiveness in applying pressure to bleeding areas. The disadvantage is that when it is not placed in a sterile bag, it must be removed by relaparotomy. It should be kept in mind that adhesions may occur in case of contact of the intestines with direct compress, as in our case. Although Gutierez et al. reported that after the removal of the packaging after 24-48 hours, hemorrhage control was achieved in $100 \%$ of cases without requiring re-operation, our case continued to bleeding [7]. The most important 
advantages of packing with the Bakri balloon include the fact that it can be inflated to the desired level, it also functions as a drain and can be removed transvaginally. Its higher cost and limited availability are disadvantages.

\section{Conclusions}

As a conclusion cardiac arrest during pregnancy is an exceedingly rare, stressful clinical condition with fatal outcomes. Timing and the whole team are highly significant in perimortem cesarian section. Pelvic packing is a quick and effective life-saving method in hemorrhage which cannot be contained with surgical and pharmacological interventions. Obstetricians should keep pelvic packing in mind and be able to perform it securely when needed.

\section{Conflict of interest disclosure}

There are no known conflicts of interest in the publication of this article. The manuscript was read and approved by all authors.

\section{Compliance with ethical standards}

Any aspect of the work covered in this manuscript has been conducted with the ethical approval of all relevant bodies and that such approvals are acknowledged within the manuscript.

\section{Authors Contribution}

$\mathrm{CH}$ manuscript writing and review

BBB and SK data collection and literature search AEC and NA conceived, designed and editing of manuscript

\section{Acknowledgments}

We would like to thank Malik Celik, MD. for illustrating Figure 1.

\section{References}

1. Wu SH, Li RS, Hwu YM. Live birth after perimortem cesarean delivery in a 36-year-old out-of-hospital cardiac arrest nulliparous woman. Taiwan J Obstet Gynecol. 2019;58(1):43-45. doi: 10.1016/j.tjog.2018.11.007

2. Katz VL. Perimortem cesarean delivery: its role in maternal mortality. Semin Perinatol. 2012;36(1):68-72. doi: 10.1053/j.semperi.2011.09.013

3. Touhami O, Bouzid A, Ben Marzouk S, Kehila M, et al. Pelvic Packing for Intractable Obstetric Hemorrhage After Emergency Peripartum Hysterectomy: A Review. Obstet Gynecol Surv. 2018;73(2):110-115. doi: 10.1097/OGX.0000000000000537

4. Jeejeebhoy FM, Zelop CM, Lipman S, Carvalho B, Joglar J, Mhyre JM, Katz VL, Lapinsky SE, Einav S, Warnes CA, Page RL, Griffin RE, Jain A, Dainty KN, Arafeh J, Windrim R, Koren G, Callaway CW; American Heart Association Emergency Cardiovascular Care Committee, Council on Cardiopulmonary, Critical Care, Perioperative and Resuscitation, Council on Cardiovascular Diseases in the Young, and Council on Clinical Cardiology. Cardiac Arrest in Pregnancy: A Scientific Statement From the American Heart Association. Circulation. 2015;132(18):1747-73. doi: 10.1161/CIR.0000000000000300

5. Whitten M, Irvine LM. Postmortem and perimortem caesarean section: what are the indications? J $R$ Soc Med. 2000;93(1):6-9. doi: 10.1177/014107680009300103

6. Ramseyer AM, Lutgendorf MA. Implementation of Low-Cost Obstetric Hemorrhage Simulation Training Models for Resident Education. Mil Med. 2019;184(1112):e637-e641. doi: 10.1093/milmed/usz098

7. Naranjo-Gutiérrez LA, Oliva-Cristerna J, RamírezMontiel ML, Ortiz MI. Pelvic packing with vaginal traction for the management of intractable hemorrhage. Int J Gynaecol Obstet. 2014;127(1):21-4. doi: 10.1016/j.ijgo.2014.04.007 